\section{The Management Centre Innsbruck Keeping one step ahead with algae innovation}

Elsbeth Heinzelmann, science + technology journalist

Abstract: $\mathrm{MCl}$ - Management Center Innsbruck is an innovative breeding ground for making algae biotechnological concepts economically attractive, useful for society and environmentally friendly. For biotechnet Switzerland there are solid reasons for entering into a strategic partnership with the $\mathrm{MCl}$ in the domain of bioresource technologies at the international level.

\section{Keywords: Algae cultures · Chlamydomonas reinhardtii ·} Desensitization to allergies · Edible vaccines · Microalgaebased food and feed products - Molecular algae biotechnology - Terrestrial, airborne and lichen algae

In the kingdom of the pharaohs, green had a positive force, serving as a symbol of regeneration and rebirth, and the renewal and restoration of depleted energy. For us today green algae are a promising source of biofuels and a sustainable supplier of a range of higher-value products, whether foods or specialty feeds, chemicals or cosmetics and pharmaceuticals.

\section{Green Alga - ‘Guinea Pig' for Research}

The ideal candidate for basic research on the molecular biotechnology of microalgae is the green alga Chlamydomonas reinhardtii. Since the Food and Drug Administration (FDA) classifies this organism as GRAS (Generally Regarded As Safe), it is also the favourite model organism at the Management Centre Innsbruck (MCI). At this university centre, founded in 1995/96, researchers are working on optimizing methods to improve the reproducibility and yield of transgenic expression.

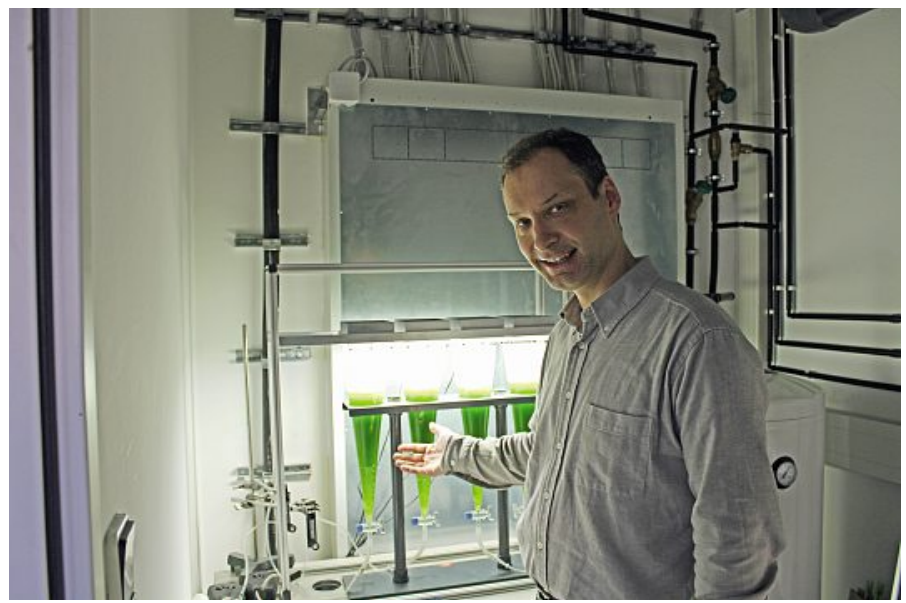

As Professor Christoph Griesbeck, Head of Biotechnology and Study Director comments, microalgae-based systems have the potential to combine the advantages of plants with features of microorganisms, thus offering an alternative to gene farming. Photo Elsbeth Heinzelmann.
As an example, and in collaboration with the University of Salzburg, the group provides allergens in the green algae $C$. reinhardtii. With the complete algae cultures the researchers are able - using the produced allergens - to treat mice by a specific immunotherapy that causes their bodies to become desensitised to allergies. "For the time being we are working on algae that produce the major allergen from birch pollen in their cells", explains Christoph Griesbeck. "The possibility to administer the whole cell culture may render the complex and cost-intensive purification unnecessary in the future. On the other hand, the given algae components could also serve as an adjuvant that increases the therapeutic effect."

To use the microalgae for biotechnological purposes, they consider the complete process chain from cultivation to isolation and analysis. Only the most promising microalgae strains identified by bioactivity screenings of biobanks are analyzed for identification of optimized growth parameters. This will result in the creation of systems like the economic photo-bioreactor with optimized light, nutrient input and flexible regulation for induction of biomass and/or induction of active agent production. The downstream processing has improved dramatically with the introduction of new methods for microalgae cell disruption and extraction of active agents.

\section{Algae Biotechnology for Novel Vaccines}

To exploit the potential of algae in order to realize products of social benefit, the MCI is actively researching molecular algae biotechnology. This involves the development and optimization of promotors and reporters, as well as the transgenic expression of allergens and edible vaccines. Concerning the latter, C. reinhardtii, a single-cell green alga measuring 10 micrometres in diameter widely distributed in soil and fresh water - offers established methods for transformation, markers and reporters. Its ability to express proteins with biopharmaceutical or biotechnological

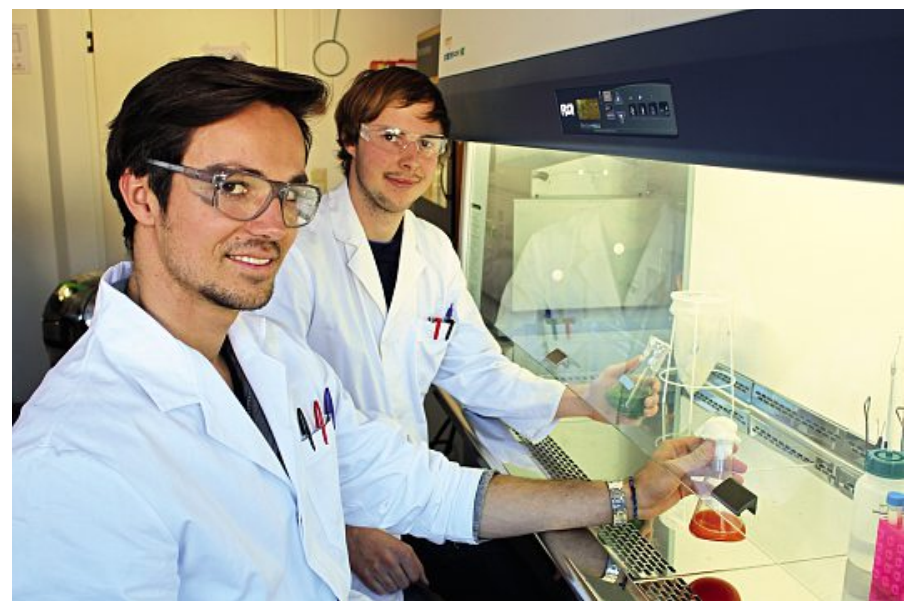

Students examine algae strains under laminar flow as they change colour from green to orange depending on the cultivation conditions. This shows that we need to handle the cultivation with great care as it has a considerable influence on product formation. Photo Elsbeth Heinzelmann. 
relevance, such as antibodies, enzymes or antigenic peptides, has been demonstrated in a number of cases.

The production of biotechnologically relevant recombinant proteins is a fast-growing market. However, current expression systems are very expensive in terms of cultivation and reconditioning. $C$. reinhardtii offers an alternative as it can be ingested as a pellet and thus serve as an oral vaccine. To increase the hitherto unsatisfactory expression rates by heat induction, the researchers use synthetic heat shock components.

In a first step, the group led by Professor Christoph Griesbeck, Head of Biotechnology \& Study Director, cloned a plasmid DNA construct containing HSE8x in connection with the Rbcs2 promotor internal to $C$. reinhardtii, and Renilla luciferase as a reporter gene. After reproduction in $E$. coli the plasmids were transformed with the glass bead technique into the $C$. reinhardtii stem $302 \mathrm{cw} 15 \arg 7$ and selected by arginine auxotrophy. Using these synthetic heat shock components, repeated eightfold in combination with Rbcs 2 after heat induction, the researchers achieved expression rates of transgenes that are three times higher compared to conventional promoters. The studies, performed in collaboration with the Humboldt University in Berlin and the FH Campus in Vienna, show that it is possible, in principle, to use $C$. reinhardtii as a production organism for biotechnically and pharmaceutically relevant proteins, for example oral vaccines. "The application of algal systems to edible vaccines is increasingly gaining interest", comments Christoph Griesbeck. "Aside from biopharmaceuticals, an additional application of algae for pharmaceutical products could involve novel metabolites improved by metabolic engineering."

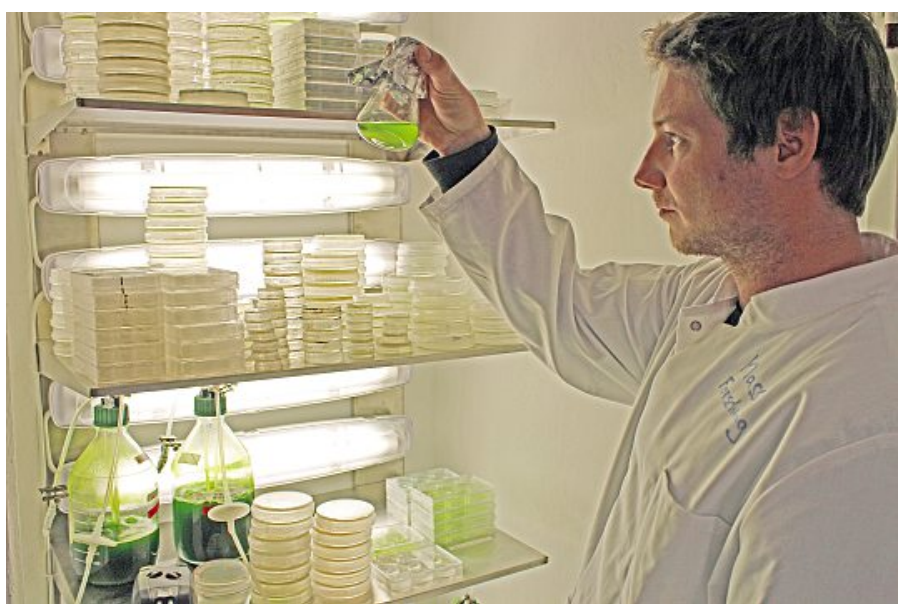

Continuous control of cultivation is necessary. As a model organism the unicellular green alga Chlamydomonas reinhardtii is one of the best studied organisms and provides established methods for transformation, markers and reporters. Its ability to express proteins with biopharmaceutical or biotechnological relevance such as antibodies, enzymes or antigenic peptides, has been demonstrated many times. Photo Elsbeth Heinzelmann.

\section{In Search of Novel Compounds}

Another field of research at the MCI is the extraction of natural substances from algae. These activities range from the screening of algae collections and the development of cultivation screenings, via the optimization of cultivation parameters and the screening of substances for pharmaceutical, cosmetic and food technological applications to the material and energetic recovery of biomass. Since the MCI is part of the Open University Innsbruck concept, the partner of choice in this domain is the Institute of Botany at the University of Innsbruck. Its collection of algae dates back to the late 1950s and comprises about 1500 strains, mostly from alpine areas of Central Europe, with a focus on terrestrial, airborne and lichen algae. In 1974 the collection was expanded by incorporating the algae collection of Swiss botanist Wilhelm Vischer, a former lecturer at the University of Basel.

Microalgae as a group of photosynthetic organisms are particularly interesting for MCI researchers: they offer valuable substances and low-cost growth in the presence of light, thereby offering untapped potential. Their activities focus on unsearched biobanks, especially a collection of terrestrial algae: the scientists assume that they would be able to exploit innovative active agents, for instance for the pharmaceutical industry.

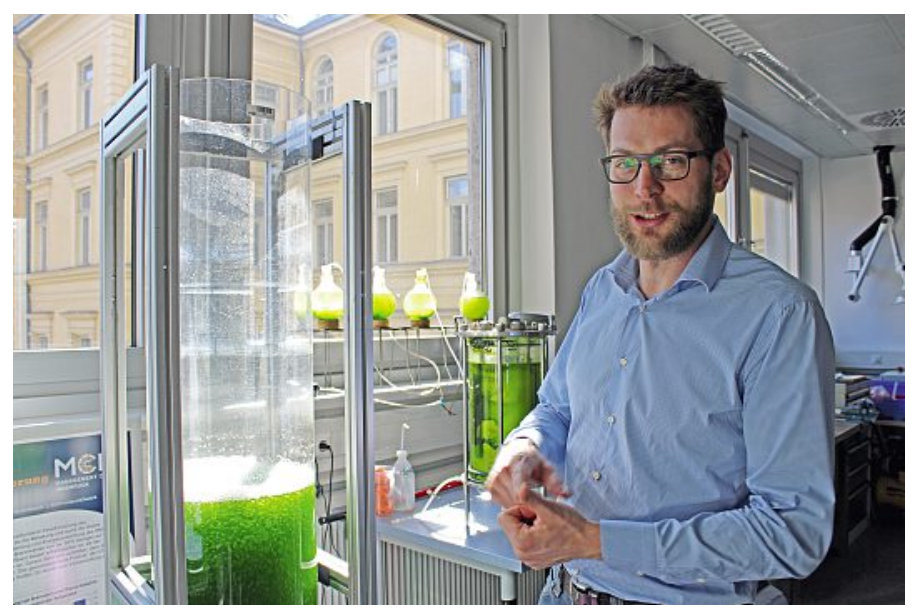

Marco Rupprich, Study Director in environmental engineering, energy management and process engineering and Managing Partner at ionOXess $\mathrm{GmbH}$, explains the algae reactor used for cultivating algae with a working volume of up to 100 litres. Photo Elsbeth Heinzelmann.

\section{Central European Lichen Algae in the Limelight}

Sometimes the most interesting discoveries are made right on our doorstep: In their observations in Tyrol and Upper Austria, the researchers discovered lichens attached to tree trunks. This fungus is inhabited by microscopic algae. It's a win-win situation: the algae enjoy a stable environment, the fungus benefits from highly enriched materials produced by the algae from carbon dioxide and solar light. Nevertheless, if the fungus grows excessively, it takes up too much sunlight. The scientists conclude that, as a protective measure, the symbiotic algae produce substances that inhibit or restrict the growth of the fungus. "There is strong evidence to indicate that these substances may also inhibit the spread of fungi", speculates Christoph Griesbeck. We already know that algae have antibacterial effects, but we do not yet understand the exact functionality of this process. Also, the science community is investigating new ways of promoting algae in order to reduce antibiotic use.

Naturally algae come to mind in the food chain: Salmon, for instance, is a great source of omega 3 essential fatty acids. "Nevertheless, salmon do not produce this substance but obtain it from what they eat, i.e. smaller oily feeder fish that consume omega-3-rich algae", comments Christoph Griesbeck. "We are therefore investigating how, and in what quantities, algae are able to produce fatty acids that are so precious for our health."

Algae technology is taken very seriously by the European Commission's Joint Research Centre (JRC): In the context of its recently developed Bioeconomy initiative, microalgae represent an emerging biological resource of great importance for potential applications in different fields. Microalgae-based molecules show specific advantages over their synthetic and traditional counterparts that make microalgae a commercially viable source 
for the food sector. The JRC report reveals that, in the experts' opinion, the EU has the potential to become the market leader in microalgae-based food and feed products in the coming decade.

\section{Strengthen the Network for an Algae Strategy}

There are compelling arguments for strengthening the network in algae research activities: Algae grow fast, can double every few hours and be harvested every day. When growing in sunlight, algae absorb carbon dioxide and release oxygen, the air we breathe. There is no competition between algae and agriculture, as algae usually use land that is often unsuitable for traditional agriculture. Microalgae can offer a high protein and oil content, maybe to produce either biofuels or animal feeds or both. Even in the sea it is possible to grow macroalgae in the form of seaweeds, their sugars being converted into biofuels and chemicals. Algae purify wastewaters for instance in municipal waste waters, animal wastes and industrial effluents. Algal biomass can be used as an energy source. The plant itself produces different materials like plastics, chemical feedstocks, lubricants, fertilizers and cosmetics. And algae are not picky: they grow in nearly any climate in a multitude of production systems, from ponds to photobioreactors or fermenters, creating a wide range of jobs from research to engineering, from construction to farming, from marketing to financial services.

"The excellent knowledge available at the MCI prompts us to strengthen our existing relationships with the research group there, profiting from available synergies and achieving results more rapidly and efficiently", explains Professor Daniel Gygax, President of biotechnet Switzerland and the NTN Swiss Biotech. "Initial contact has been made with relevant partners at ZHAW Wädenswil and HES-SO Wallis/Valais. In the coming months we shall be creating platforms with defined tasks and common projects in the domain of the bioresource technologies for in vitro diagnostics and antibiotics at the international level." The enhanced cooperation between Tyrol and Switzerland also gives young people access to exchange studies in the area of Bachelor and Master Degrees, and opens up new possibilities in high-level training - all in all a win-win situation for both research partners.

www.mci.edu

www.biotechnet.ch 